\title{
Smarte Persönliche Assistenten gestalten
}

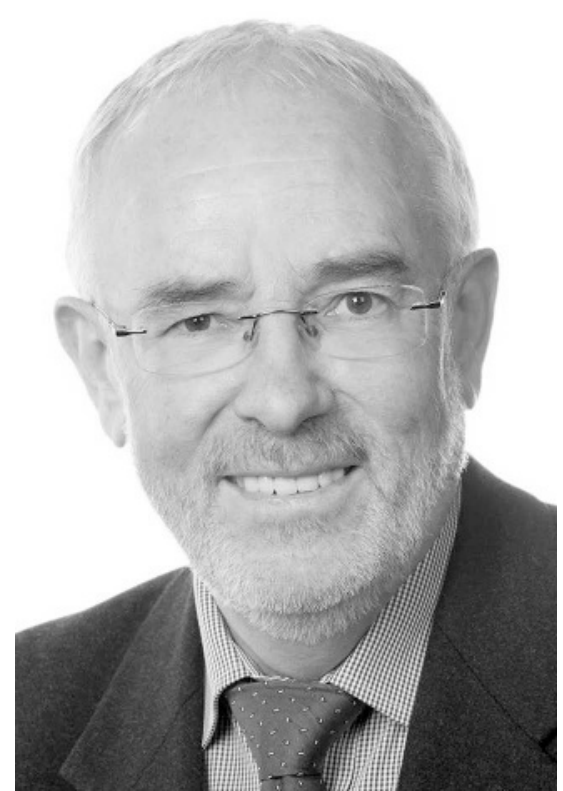

Ziele des Datenschutzrechts lassen sich vielfach nur umsetzen, wenn sie in Technik implementiert sind. Diese Erkenntnis hat schon seit langer Zeit Eingang in die Forderungen nach Datenschutz durch Technik, Systemdatenschutz oder „Privacy by Design" gefunden. Diese Forderung hat jetzt sogar die Datenschutz-Grundverordnung anerkannt. In Art. 25 Abs. 1 fordert sie, dass „der Verantwortliche sowohl zum Zeitpunkt der Festlegung der Mittel für die Verarbeitung als auch zum Zeitpunkt der eigentlichen Verarbeitung geeignete technische und organisatorische Maßnahmen - wie z. B. Pseudonymisierung - trifft, die dafür ausgelegt sind, die Datenschutzgrundsätze wie etwa Datenminimierung wirksam umzusetzen und die notwendigen Garantien in die Verarbeitung aufzunehmen, um den Anforderungen dieser Verordnung zu genügen und die Rechte der betroffenen Personen zu schützen“. Diese notwendige Vorgabe schwächt die Datenschutz-Grundverordnung jedoch selbst, indem sie zum einen die Forderung nur an den Verantwortlichen richtet, nicht aber an den Hersteller von technischen Systemen, obwohl der Verantwortliche in den meisten Fällen davon abhängig ist, was der Hersteller ihm anbietet. Hersteller werden in Erwägungsgrund 78 nur „ermutigt, ... das Recht auf Datenschutz bei der Entwicklung und Gestaltung der Produkte, Dienste und Anwendungen zu berücksichtigen“. Zum anderen stellt Art. 25 Abs. 1 diese richtige Forderung unter den Vorbehalt, dass der Verantwortliche auch den Stand der Technik, die Implementierungskosten und die Art, den Umfang, die Umstände und die Zwecke der Verarbeitung sowie die unterschiedliche Eintrittswahrscheinlichkeit und Schwere der mit der Verarbeitung verbundenen Risiken für die Rechte und Freiheiten natürlicher Personen berücksichtigen soll.

Das größte Problem ist jedoch, dass die Datenschutz-Grundverordnung diese Forderung - mit Ausnahme des Beispiels der Pseudonymisierung - nicht konkretisiert. Einfach nur die Forderung zu stellen, technische Systeme nach dem Grundsatz eines „Privacy by Design" zu gestalten, hilft in der Praxis nicht weiter. Sie wird von entgegenstehenden Gestaltungszielen wie Performanz, Flexibilität oder Personalisierung zur Seite geschoben. Fordern Datenschutzbeauftragte, in der Produktentwicklung Art. 25 Abs. 1 DSGVO einzuhalten, werden entweder die zu berücksichtigenden entgegenstehenden Belange geltend gemacht oder die Notwendigkeit einer bestimmten Gestaltungsvariante bestritten. Aufgrund der Abstraktheit der Forderungen kann der Datenschutzbeauftragte jedoch nie nachweisen, dass diese Vorschrift eine bestimmte Gestaltung fordert. Auch hier gilt die Erfahrung, dass überall da, wo das Recht normative Spielräume eröffnet, letztlich soziale, politische und wirtschaftliche Macht eindringt und einseitige Ergebnisse durchsetzt.

Selbst wenn ein Entwickler gutmütig und bereit wäre, ein Techniksystem datenschutzgerecht zu gestalten, lässt ihn diese Vorschrift im Stich. Nicht jedes Datenschutzproblem lässt sich mit der Pseudonymisierung der personenbezogenen Daten lösen. Andere Hinweise gibt die Vorschrift aber nicht. Woher soll der Entwickler Anregungen und Unterstützung für die datenschutzgerechte Gestaltung bekommen? Der Verweis auf die Einhaltung der „Datenschutzgrundsätze, wie etwa Datenminimierung" hilft oft auch nicht weiter, weil das Gebot der Datenminimierung vom Zweck abhängig ist, für den die Daten verarbeitet werden. Wenn der Verantwortliche z. B. sein System personalisieren will, dann ist für diesen Zweck u. U. eine umfassende Profilierung aller Nutzer notwendig und die Datensammlung muss nicht weiter minimiert werden.

„Privacy by Design“ ist dringend notwendig und auch möglich, aber schwierig und aufwändig. Hierfür ist es notwendig, die rechtlichen Ziele mit anderen Designzielen zusammenzuführen und zu priorisieren. Gerade durch Gestaltung des Systems ist zu versuchen, mehrere Ziele zugleich umzusetzen und so zu harmonisieren. Für wiederkehrende Gestaltungsprobleme können Muster für Gestaltungsanforderungen und Gestaltungsentwürfe helfen, die Anforderungen des Datenschutzes und anderer Gestaltungsziele zu berücksichtigen. Auf sie kann ein Entwickler in den unter- 
schiedlichen Phasen des Entwicklungsprozesses zurückgreifen.

Diese Gestaltungsprobleme stellen sich in besonderer Dringlichkeit bei Smarten Persönlichen Assistenten. Sie verbinden Möglichkeiten der Künstlichen Intelligenz, insbesondere der Spracherfassung und -erkennung und des Maschinenlernens, des Ubiquitous Computing mit Kontexterfassung und Kommunikationsfähigkeit sowie der Personalisierung. Sie dringen tief in die Gesellschaft ein und sind in vielen Wohnungen und Büros zu finden, sind Bestandteile moderner Automobile und mit den Betriebssystemen von Computern und Smartphones verbunden. Smarte Persönliche Assistenten besitzen aufgrund ihrer vielfältigen Verwendungsmöglichkeiten eine starke Attraktivität, aber aufgrund der Verarbeitung von Daten mit hoher Aussagekraft auch ein hohes Risiko für die informationelle Selbstbestimmung. Sie sind geeignete Beispiele für Zielkonflikte zwischen Dienstleistungsqualität und Rechtsverträglichkeit und stellen die Entwickler von Anwendungen Smarter Persönlicher Assistenten vor schwierige Herausforderungen.

Um dazu beizutragen, diese zu bewältigen, ist es sinnvoll, die Instrumente der Anforderungs- und Entwurfsmuster für die Gestaltung Smarter Persönlicher Assistenten nach dem Prinzip des „Privacy by Design“ zu erproben. Dies war Ziel des DFGForschungsprojekts „Anforderungs- und Entwurfsmuster zur rechtsverträglichen und qualitätszentrierten Entwicklung kontextsensitiver Applikationen“ (AnEkA) des Wissenschaftlichen Zentrums für Informationstechnikgestaltung (ITeG) der Universität Kassel. Die Beiträge im Schwerpunkt dieses Heftes stellen wesentliche Ergebnisse dieses Projekts vor und verbinden sie mit weiteren Beiträgen zu Gestaltungsfragen von Smarten Persönlichen Assistenten.

Der einleitende Beitrag von Thies, Knote, Jandt und Söllner untersucht die konfliktären Anforderung der Dienstleistungsqualität und der Rechtsverträglichkeit für die Gestaltung Smarter Persönlicher Assistenten und stellt Räume und Methoden für die Lösung dieser Konflikte vor. Der folgende Beitrag von Dickhaut, Janson, Roßnagel und Leimeister zeigt, wie Anforderungsmuster widersprüchliche Anforderungen systematisieren und ihre Harmonisierung vorbreiten können. Dickhaut, Thies und Janson zeigen, wie Technikgestaltung für Smarte Persönliche Assistenten sowohl die Dienstleistungsqualität verbessern als auch Rechtsverträglichkeit erreichen und das Gestaltungswissen für immer wiederkehrende Probleme in Entwurfsmustern kodieren kann. Wie die Anforderungs- und Entwurfsmuster sich in der Evaluation durch eine Simulationsstudie bewährt haben und welche Gestaltungsvorschläge durch diese Evaluation gewonnen werden konnten, berichten schließlich Thies, Dickhaut, Janson, Roßnagel, Leimeister und Söllner. Der Beitrag von Hobert und Berens, Universität Göttingen, untersucht, wie Chatbot-basierte Lernsysteme datensparsam entwickelt und eingesetzt werden können. Geminn, Szczuka, Weber, Artelt und Varonina vom Forschungsprojekt IMPACT analysieren, welcher spezifische Gestaltungsbedarf zum Schutz von Kindern zu beachten ist, wenn diese Smarte Sprachassistenten nutzen.

Alle Beiträge kommen - auf unterschiedlichen Wegen und mit unterschiedlicher Gewichtung - letztlich zu dem Ergebnis, dass Smarte Persönliche Assistenten besondere, konfliktäre Gestaltungsanforderungen aufweisen und dass Datenschutz in der Praxis umgesetzt werden kann, wenn diese Anforderungen systematisch erkannt und durch Gestaltungslösungen aufeinander abgestimmt werden.

\section{Alexander Roßnagel}

